\title{
An Energy Management System for a Smart Office Environment
}

\author{
Cristina Rottondi*, Markus Duchon ${ }^{\dagger}$, Dagmar Koss ${ }^{\dagger}$, Giacomo Verticale*, and Bernhard Schätz ${ }^{\dagger}$ \\ * Dipartimento di Elettronica, Informazione e Bioingegneria, Politecnico di Milano, Piazza Leonardo da Vinci, 32, Milan, Italy \\ \{cristina.rottondi,giacomo.verticale\}@polimi.it, \\ $\dagger$ fortiss GmbH, Guerickestrasse 25, 80805 Munich, Germany, \{duchon,koss,schaetz\}@ fortiss.org
}

\begin{abstract}
The evolution of the electricity grid towards the smart grid paradigm is fostering the integration of distributed renewable energy sources in Smart Buildings: a combination of local power generation, battery storage and controllable loads can greatly increase the energetic self-sufficiency of a Smart Building, enabling it to operate in islanded mode or to participate in an Automatic Demand Response framework, thus taking advantage of time-variable tariffs to achieve economical savings.

This paper proposes an energy management system specifically tailored for a Smart Office building, which relies on actual data and on forecasting algorithms to predict the future patterns of both local energy generation and power loads. Performance is compared to the optimal energy usage scheduling, which would be obtained assuming the exact knowledge of the future energy production and consumption trends, showing gaps below $10 \%$ w.r.t. the optimum.
\end{abstract}

Index Terms-Smart Office Building; Real-time Load Scheduling; Photovoltaic Generation; Battery Storage;

\section{INTRODUCTION}

The novel smart grid concept is not only revolutionizing the electricity grid infrastructure, but also incentivizing awareness of a more sustainable energy utilization: "green" solutions for residential and commercial buildings have been investigated with the aim of increasing the diffusion of renewable energy sources and reducing carbon footprints [1], [2]. However, the inherently intermittent production patterns of renewables (such as solar and wind energy) increase the unpredictability of the overall power availability, thus rising power balancing issues in the management of the smart grid [3]. To cope with such challenges, the "Smart Building" paradigm proposes to integrate distributed generation plants, storage capabilities and electrical loads. Each Smart Building can be managed by a dedicated control system and operates either in a gridconnected fashion or in islanded mode. In the former, the Smart Building can either absorb or inject power in the grid, according to its needs. The latter implies complete energetic autonomy of the Smart Building, which must rely exclusively on local generation and storage to satisfy the power request of the electrical loads without receiving any feeding from the grid in case of shortage, nor injecting power surpluses into the grid in case of excessive production.

The choice of the operational state of the Smart Building affects both the management of the storage capabilities and the scheduling of the power loads, in case they exhibit some malleability (e.g. deferrable loads such as the recharge of the batteries of electric vehicles or electronic devices, or tunable loads such as cooling and heating systems). Several management policies have been investigated, mostly aimed at the minimization of the operational costs in presence of timevariable energy tariffs (assuming to operate in grid-connected mode) [4], or of the marginal costs when operating in islanded mode [5].

This paper proposes an energy management system for a Smart Office Building equipped with a photovoltaic plant, a small storage bank and a set of loads (either non-deferrable or deferrable). The management system works in real-time based on energy production and consumption forecasting algorithms, which exploit the following peculiarities of the Smart Office ecosystem:

- with respect to residential buildings, in which power consumption usually exhibits peaks in the early morning and during the evenings, in a Smart Office the production pattern of the photovoltaic plant is better aligned with the peak consumption periods, which usually occur during the day;

- heating, cooling, and lighting consumption can be forecasted according to the utilization schedules of the rooms (e.g., the usage of conference rooms is mostly preplanned by means of a booking mechanism, the occupation of offices depends on the travelling and time-off patterns of the employees);

- deferrable loads such as the battery recharge of laptops and mobile devices can be planned according to the periods in which the devices are plugged-in at the working stations, which can be declared in advance by the device owners according to their daily working schedule.

The proposed management system defines the periods in which the Smart Office operates in islanded or grid connected mode. In the latter case, it also defines the amount of energy to be absorbed/injected into the grid based on the electricity tariffs. To do so, the energy manager solves a Mixed Integer Linear Program (MILP) at regular intervals, which takes as input both the actual energy production/consumption data and forecasts about the future production/consumption patterns. 
The remainder of the paper is structured as follows: Section II provides a brief overview of the related work, whereas Section III introduces the general framework of the Smart Office Building, the energy production/consumption forecasting algorithms and the MILP executed by the management system. Section IV compares the results obtained by the proposed system to the performance achieved by running the MILP under the assumption of full knowledge of the future energy production/consumption trends. Section V concludes the paper.

\section{RELATED WORK}

Several optimization methods for the management of a Smart Building have been recently proposed by the scientific community. Solutions tailored for both residential and commercial environments have been discussed, with strategies ranging from day-ahead to real-time planning. Guan et al. [2] design a MILP for the minimization of gas and electricity bills of a university campus building equipped with a controllable combined heat-power system, battery storage and a photovoltaic plant. The program is applied both under the assumption of a deterministic scenario or of a "scenario tree", where uncertainty about future power usage is taken into account by means of a weighted objective function including various production/consumption patterns, each one occurring with different probabilities. Our approach also uses a linear program, but the deterministic case is compared to a real-time optimization method in which the model is solved multiple times during the optimization horizon, and scheduling decisions are dynamically updated.

More refined linear programs for the energy management of a household are provided by Bozchalui et al. [6] and Kriett et al. [4], who model the behavior of individual electrical appliances and combine multiple objectives such as the minimization of energy consumption, energy costs, carbon emissions, and peak load. Our proposed energy management system also considers different objective functions.

A few recent works have specifically addressed the peculiarities of a Smart Office environment: Stojanovic et al. [7] focus on energy saving strategies for lightening management based on room occupancy. Real testbeds deployed in office buildings aimed at the development of self-sustained distributed energy systems are described in [8]-[10]. The results presented in this paper have been obtained based on data provided by the "Smart Energy Living Lab" located at the fortiss premises [10].

\section{The SMART OfFICE ENVIRONMENT}

The fortiss Smart Energy Living Lab is envisioned as a Smart Building (i.e. a system that can produce, consume, and store energy) and is an example for adaptive control and responsive behavior. Based on the current and predicted generation of renewable energy and on the available storage capacity, it is possible to control office appliances in order to achieve a better utilization of energy. Imagine a sunny day, which leads to an overproduction of the solar energy generation. Once the batteries of the storage bank are fully charged, the surplus could be used to cool down the server room more than usual. Hence, the server room has not to be cooled down for a longer period of time. Alternatively, taking the current energy prices into account, the excess energy can be feed into the network to achieve an economic profit. Another scenario related to dynamic energy prices is the switching between islanded and grid connected mode. Again, mechanisms for efficient energy use can be used to avoid prolonged high price periods.

In the following we will summarize the general framework of the fortiss Smart Energy Living Lab [11] before explaining the components and algorithms used to predict the future energy generation/consumption and to schedule the Smart Building operations.

\section{A. General Framework}

For the Smart Energy Living Lab a flexible, extensible and lightweight architecture is used, which follows a layered and component based approach to ensure scalability, flexibility and extensibility. An overview of the system is illustrated in Figure 1. Starting at the bottom, several sensors and actuators are connected to the Server Application, or middleware, respectively. The sensor and actuator layer of the middleware supports different protocols like IEC61850 via Modbus, ZigBee, and EnOcean to exchange information and control signals with different sensors and actuators from both the home automation and the energy domain. This enables the system to provide real time monitoring and control capabilities for the photovoltaic installation, the backup batteries, air condition, blinds, lights, power plugs, window sensors, humidity, temperature, brightness, and powermeters. For additional details regarding the system middleware, the reader is referred to [10]. Software components within the system layer of the Server Application enable the management of users, their roles, associations to rooms and personal profiles as well as the modeling of Smart Building environments in terms of assigning devices to rooms and these to floors or buildings respectively. This layer includes a central registrar to enable the integration of additional sensors and actuators in a plug and play manner. Corresponding observation data and control capabilities can be made available via a REST or WebSocket API on different devices and clients. Besides the utilization of real time data all information is stored in, or retrieved from a database. In addition, a rule system at the application layer of the middleware is used to observe the current status and under appropriate conditions to issue commands e.g. to maintain a defined brightness level. Nevertheless, a detailed description of the rule system is out of scope of this work, as well as another component which extracts knowledge out of historical data.

\section{B. Energy Production and Consumption Forecast Algorithms}

For the determination of optimized schedules to achieve economic savings taking into account operational mode, load shifting and variable energy tariffs, forecasts of both the local energy generation and consumption are essential. In this work 


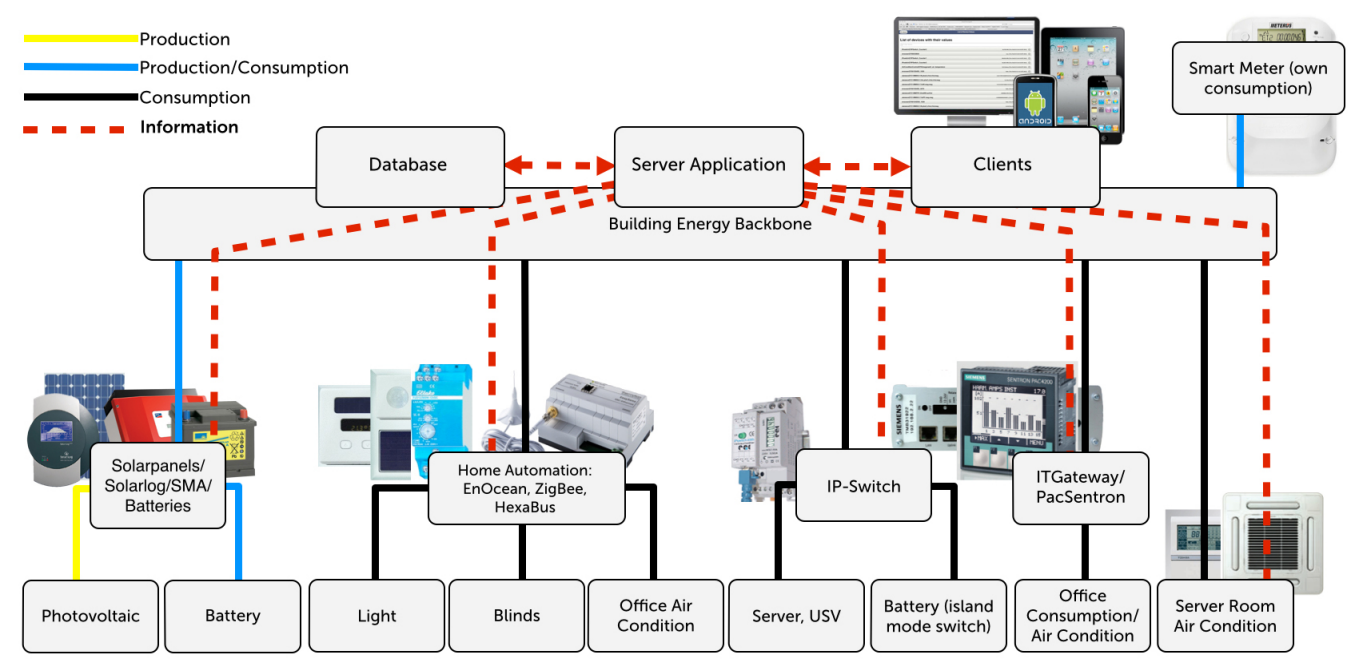

Fig. 1: Overview of the Smart Energy Living Lab

the forecasts are generated at midnight for the next 24 hours with a granularity of 15 minute intervals.

Generation Forecast: The generation forecast utilizes the OpenWeatherMap API [12], which provides weather forecasts for three hour periods. Available information include sunrise and sunset times, a weather condition code, and the percentage of sky coverage due to clouds. Since energy generation using a photovoltaic installation depends on the time of the year, we assume due to simplicity reasons a parabolic peak production for each month $(-5, \ldots, 6)$ with a maximum in July:

$$
P_{\text {max }}(\text { month })=-75 \cdot(\text { month })^{2}+P V_{\text {peak }} \quad[W]
$$

where $P V_{\text {peak }}$ indicates the peak production of the installed plant. The weather condition code $\gamma(t)$ (e.g. clear sky, scattered clouds, or moderate rain) and the percentage of sky coverage $\phi$ form a weather factor $\omega(t)=\frac{\gamma(t)+\phi(t)}{2}$ in the range $[0, \ldots, 1]$ (e.g. 0.0 for heavy rain and 1.0 for clear sky). To model an ideal generation we utilize two Gaussian distributions and select the minimum value at desired points in time $i$ between sunrise and sunset with a granularity of 15 minutes. Such points are mapped between $-\mathrm{e}$ and e as steps $s_{i}$. Before sunrise and after sunset the generation forecast $G_{f c}$ is zero, otherwise it is calculated as follows:

$$
G_{f c}(t)=\omega(t) \cdot P_{\max }(\text { month }) \cdot \min \left(\frac{1.0}{\mathrm{e}^{0.3 \cdot(i)^{2}}}, \frac{1.0}{2 \cdot \mathrm{e}^{0.75 \cdot(i)^{2}}}\right)
$$

Figure 2 illustrates the results of the actual (solid, blue) and predicted (dashed, green) generation.

Consumption Forecast: We applied a triple exponential smoothing model provided by openforecast [13] to predict the power consumption, since seasonal models are supposed to be a simple but feasible approach for short-term electricity demand [14], [15]. Initial tests have shown that the following parameterization generates sufficiently adequate results: TripleExponentialSmoothingModel $(0.7,0.1,0.2)$ which correspond to the weight of recent data, trend, and seasonality, respectively. The basis for these models are time series, where we use the historical consumption data for the past six same days (either working or high days). If we utilized information from the last successive days, the strong differences between workdays and weekends would distort the forecast values. Figure 3 illustrates the results of the actual (solid, blue) and predicted (dashed, green) consumption, where the difference in the power demand of workdays and the weekends becomes evident. Currently we are working on the integration of additional calendar information regarding the booking of the conference rooms including the expected participants and the type of the meeting. In our approach we will subtract the consumption of the respective conference rooms, which are also monitored, from the overall consumption used for the described demand forecast. In a second step, predefined consumption profiles for each conference room will be added to the calculated forecast for the period of the reservations of the corresponding room, e.g. when it is booked for a presentation from $3 \mathrm{pm}$ until $5 \mathrm{pm}$. Finally, we will compare and evaluate our approach with others, however, this is beyond the focus of this work.

\section{Real-time Energy Manager}

The energy management algorithm assumes that the optimization horizon is divided in $T$ epochs of fixed duration (e.g. in the order of minutes) and works under the following assumptions:

- when operating in grid-connected mode, the Smart Building absorbs/inject energy directly from/to the grid, whereas the backup battery is automatically recharged at constant rate until full charge level and cannot be discharged ${ }^{1}$;

- when operating in islanded mode, the Smart Building absorbs/inject energy at arbitrary rate from the backup battery. In case of excess in energy production, which cannot be absorbed by the backup battery, surpluses are

\footnotetext{
${ }^{1}$ This assumption is made to meet the constraints imposed by the fortiss Smart Energy Living Lab.
} 


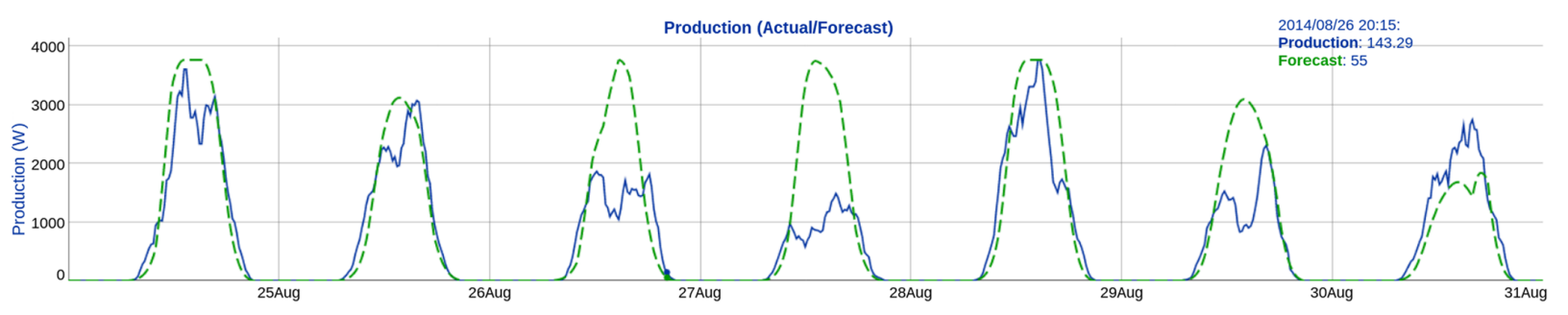

Fig. 2: Comparison of actual and predicted production

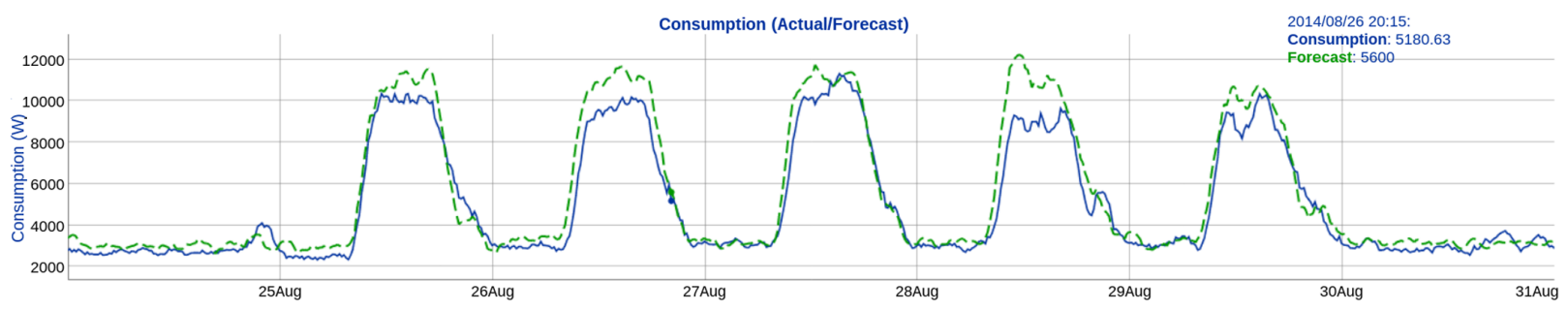

Fig. 3: Comparison of actual and predicted consumption

dissipated. Conversely, in case of production deficits, which cannot be fully compensated by the energy stored in the backup battery, loads must be shed (e.g. by switching off non-critical appliances);

- the duration of plug-in periods of rechargeable electronic devices is specified by the owners at the moment of plugging the device. Alternatively, these periods could be enforced by using switchable sockets controlled by the system. The recharge process can possibly experience intermediate interruptions. Recharge is mandatory if the current state of charge of the device battery is below a given threshold specified by the user.

Whenever a new epoch $i$ starts, the energy manager receives the energy production/consumption forecasts computed by the algorithms presented in Section III-B, the actual amounts of energy generated and consumed in the previous epochs $1, \ldots, i-1$, the current state of charge of the backup battery and of the batteries of the electronic devices actually plugged for recharge. The expected plug-in periods of the electronic devices can be either randomly generated or computed according to historical data. The energy manager then runs a MILP to schedule the energy usage for the current epoch, which is defined as follows:

\section{Inputs:}

- $p_{i}$ : forecasted energy production of the photovoltaic plant for epochs $i, \ldots, T$;

- $c_{i}$ : forecasted energy consumption of non-deferrable loads for epochs $i, \ldots, T$;

- $e p_{i} / e s_{i}$ : energy purchasing/selling price for epochs $i, \ldots, T ;{ }^{2}$

- $B$ : actual state of charge of the backup battery at the end of epoch $i-1$;

\footnotetext{
${ }^{2}$ Note that the energy tariff can be either known in advance or dynamically adjusted. In the latter case, energy prices are forecasted according to historical knowledge and then updated epoch by epoch according to the actual values.
}

- $R$ : recharge rate of the backup battery (only for gridconnected mode);

- forecasted/actual plug-in periods $\left(w_{i}^{j}\right)$, state of charge $\left(B w^{j}\right)$, and battery recharge rate $\left(R w^{j}\right)$ of each rechargeable device $j$;

- $r_{i}^{j}$ : reward for recharge of device $j$ at epoch $i$;

Outputs:

- $x_{i}$ : choice of the operational mode (islanded $=0$ or gridconnected=1) for epochs $i, \ldots, T$;

- $y_{i}^{p} / y_{i}^{s}$ : amount of purchased/sold energy at epoch for epochs $i, \ldots, T$ ( 0 if in islanded mode);

- $z_{i}^{j}$ : schedule of the recharge periods of electronic devices for epochs $i, \ldots, T$ (not in charge $=0$, in charge $=1$ );

Objective Function:

$$
\min \sum_{i=1, \ldots, T}\left(y_{i}^{p} e p_{i}-y_{i}^{s} e s_{i}-\sum_{j \in J} z_{i}^{y} r_{i}^{y}\right)
$$

The model performs a multi-objective optimization, i.e. the minimization of a weighted sum of multiple contributions, including operational costs (in terms of daily energy expenses) and rewards for the recharge of the batteries of the electronic devices above the minimal threshold. Rewards are design parameters which can be adjusted to privilege one or the other objective.

The operational mode for epoch $i$ and the recharge of the batteries of the electronic devices is then settled according to the MILP output. At the end of epoch $i$, in case of islanded operation regime the state of charge of the battery is updated according to the real energy usage trend, whereas in case of grid-connected regime operational costs are updated according to the exact amount of energy absorbed/injected from/into the grid.

\section{Energy Manager Integration}

In the following we shortly describe the integration and interaction of the energy manager into the current system. The 
presented energy management algorithm will be implemented as an OSGi bundle, whereas the execution will be carried out periodically. The interaction with the other components will be realized using RPC calls over the rabbitMQ message bus system [16]. The required input parameters are provided by the Smart Energy Living Lab using the same technology. The system periodically generates the described forecasts at midnight for the next 24 hours, although the responsible component supports requests at any time the optimization interval and the number of epochs. This enables readjustments of earlier forecasts, especially to improve the short term prediction quality. In order to control the required actuators, the existing rule system API [10] will be extended to allow the input from the energy manager. Here, the choice of the operational mode can be modeled as a list containing a timestamp referring to the epoch and an indicator for the mode. In this way, the schedule of recharge periods can be represented too. In case of updates due to differences between the forecast and actual production/consumption this list can be easily updated. The rule system already supports the functionality to switch on and off necessary devices. Switchable sockets currently consist of Hexabus [17], ZigBee and EnOcean devices. The change of the operational mode is carried out by using an IPSwitch-SGIn [18] which is already integrated.

\section{Performance Evaluation}

To assess the performance of our proposed energy management system, we tested it in the fortiss Smart Energy Living Lab. The testbed includes a photovoltaic plant with peak production of $3770 \mathrm{Wp}$, a backup battery with capacity of $6720 \mathrm{Wh}$ and recharge rate of $1200 \mathrm{~W}$, a set of nondeferrable appliances (lights, heating/cooling systems, servers and desktop computers), and six controllable plugs to which three laptops (device battery capacity of $55 \mathrm{Wh}$, recharge rate of $45 \mathrm{~W}$ ) and three mobile phones (device battery capacity of $6 \mathrm{Wh}$, recharge rate of $3 \mathrm{~W}$ ) can be connected. Recharge is mandatory until device batteries reach $65 \%$ of charge.

The scheduling horizon is a 24 hour period divided in $T=96$ epochs of 15 minute duration. The objective function minimizes the overall operational costs and rewards the recharge of the electronic devices by decreasing the energy bill by the corresponding recharging cost (i.e. $r_{i}^{j}=e p_{i}$ ). The energy prices vary according to the tariffs provided in [19], which usually exhibit the highest peaks in the morning (9-11 a.m.) and in the early evening (6-8 p.m.). Prices for selling energy surpluses by injection into the grid are obtained by multiplying the actual purchase prices by a scaling factor $\alpha=0.7$ (i.e. $e s_{i}=\alpha e p_{i}$ ).

For the sake of concision, here we limit the analysis of the numerical results to two reference week and weekend days. Figures 4 and 5 show an example of the forecasted/actual energy production/consumption patterns of the photovoltaic plant and the must-run appliances during a sunny weekend day and a partially cloudy working day, respectively. The corresponding forecasted/actual energy exchanges with the grid obtained

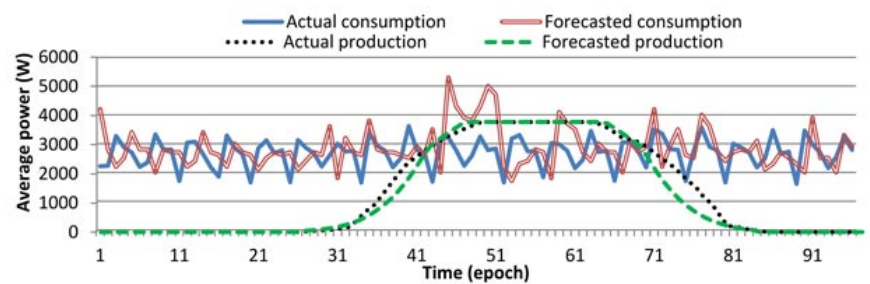

Fig. 4: Actual and predicted production/consumption daily trend (weekend)

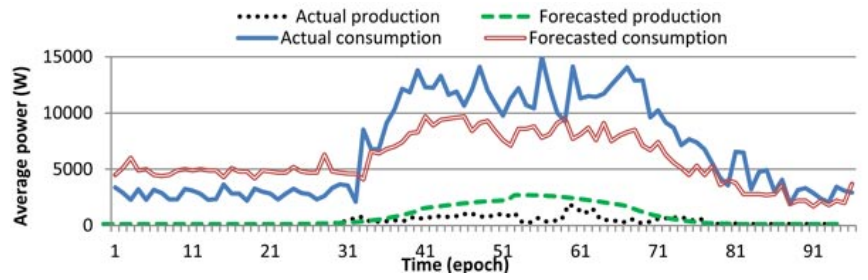

Fig. 5: Actual and predicted production/consumption daily trend (working day)

by means of the real-time energy management system ${ }^{3}$ are compared to the optimal benchmark schedules computed "a posteriori" in Figures 6 and 7. The trends depicted in Figure 6 show that during a sunny weekend, the power generated by the photovoltaic plant is sufficient to support the islanded operational mode for most of the daylight time. This also avoids to purchase energy from the grid during the peak-price periods occurring from 8 to 12 a.m. (i.e. from epoch 30 to 45). Conversely, during weekdays the photovoltaic production is not sufficient to ensure prolonged periods in islanded mode, which are thus limited to a few epochs corresponding to the highest price peaks (e.g. epochs 43 and 83, see Figure 7). In both scenarios, our proposed energy management system achieves close-to-optimum performance, with gaps of $8.2 \%$ and $0.2 \%$ w.r.t. the benchmark cost, respectively. As expected, the gap is lower for the weekday, where the flexibility in the management of the battery charge/dischrage process is lower due to the high energy consumption, which almost always considerably exceeds production, thus preventing prolonged islanded periods. It is worth noting that, in absence of storage capability and constantly operating in grid-connected mode, the overall cost would be $67.8 \%$ and $9.2 \%$ higher than the benchmark.

However, when operating in islanded mode, production surpluses or deficits may occur, e.g. in case of significant under/overestimations of the power usage/generation by the forecasting algorithms w.r.t. the actual values. In the presented results, the latter case occurs twice in Figure 7 (see epochs 43 and 83), but the impact of the power shortage is mild (below $1 \mathrm{~kW}$ ) and could easily be compensated e.g. by turning off the heating/cooling system for a few minutes.

\section{CONCLusions}

This paper describes a real-time energy management system for a Smart Office environment, which combines fore-

\footnotetext{
${ }^{3}$ Note that the running time of the AMPL/CPLEX solver on a standard desktop computer was below $1 \mathrm{~min}$ in all the considered instances.
} 


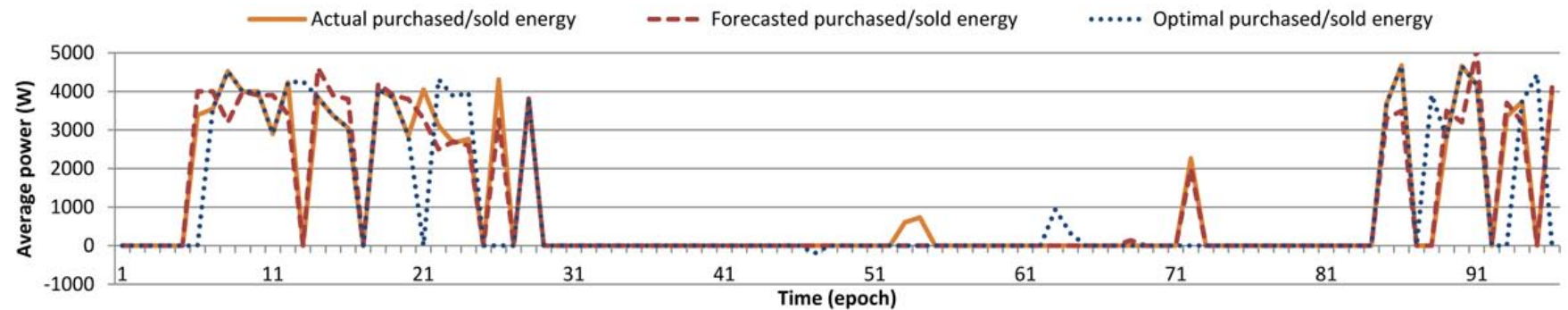

Fig. 6: Forecasted, actual and optimal daily trend of energy purchase/sell (weekend). Zero values occur during periods of islanded regime. Negative values indicate energy deficits.

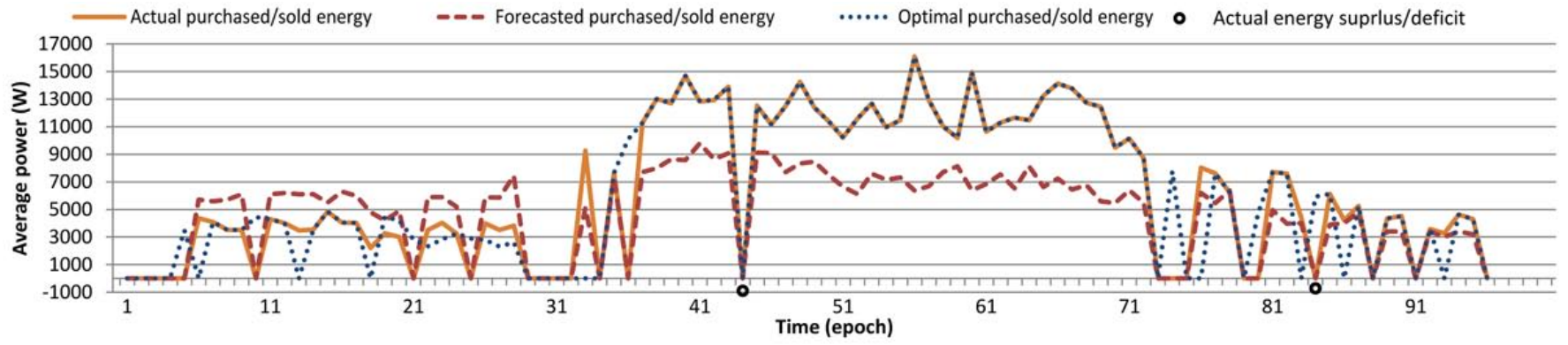

Fig. 7: Forecasted, actual and optimal daily trend of energy purchase/sell (working day). Zero values occur during periods of islanded regime. Negative values indicate energy deficits.

casting algorithms for the predictions of energy production/consumption trends with an optimizer that schedules the Smart Building operations according to the forecasted and actual energy utilization patterns, as well as to the current energy prices. Based on the presented results, we believe that the integration of our proposed system is a valid support to achieve nearly-optimal schedules of the Smart Building operational mode and to ensure significant cost savings.

\section{ACKNOWLEDGMENTS}

The work in this paper has been partially funded by Regione Lombardia under grant no. 40545387 Smart Campus as Urban Open LAbs(SCUOLA). The authors would like to thank the EIT ICT Labs for the support to build up the demonstrator.

\section{REFERENCES}

[1] J. Kleissl and Y. Agarwal, "Cyber-physical energy systems: Focus on smart buildings," in Proceedings of the 47th Design Automation Conference, ser. DAC '10. New York, NY, USA: ACM, 2010, pp. $749-754$.

[2] X. Guan, Z. Xu, and Q.-S. Jia, "Energy-efficient buildings facilitated by microgrid," Smart Grid, IEEE Transactions on, vol. 1, no. 3, pp. 243-252, Dec 2010.

[3] A. Angelis-Dimakis, M. Biberacher, J. Dominguez, G. Fiorese, S. Gadocha, E. Gnansounou, G. Guariso, A. Kartalidis, L. Panichelli, I. Pinedo, and M. Robba, "Methods and tools to evaluate the availability of renewable energy sources," Renewable and Sustainable Energy Reviews, vol. 15, no. 2, pp. 1182 - 1200, 2011.

[4] P. O. Kriett and M. Salani, "Optimal control of a residential microgrid," Energy, vol. 42, no. 1, pp. 321 - 330, 2012, 8th World Energy System Conference, $\{$ WESC $\} 2010$.

[5] H. Morais, P. Kdr, P. Faria, Z. A. Vale, and H. Khodr, "Optimal scheduling of a renewable micro-grid in an isolated load area using mixed-integer linear programming," Renewable Energy, vol. 35, no. 1, pp. $151-156,2010$
[6] M. Bozchalui, S. Hashmi, H. Hassen, C. Canizares, and K. Bhattacharya, "Optimal operation of residential energy hubs in smart grids," Smart Grid, IEEE Transactions on, vol. 3, no. 4, pp. 1755-1766, Dec 2012.

[7] N. Stojanovic, D. Milenovic, Y. Xu, L. Stojanovic, D. Anicic, and R. Studer, "An intelligent event-driven approach for efficient energy consumption in commercial buildings: Smart office use case," in Proceedings of the 5th ACM International Conference on Distributed Eventbased System, ser. DEBS '11. New York, NY, USA: ACM, 2011, pp. 303-312.

[8] M. Noritake, H. Hoshi, K. Hirose, H. Kita, R. Hara, and M. Yagami, "Operation algorithm of dc microgrid for achieving local production for local consumption of renewable energy," in Telecommunications Energy Conference 'Smart Power and Efficiency' (INTELEC), Proceedings of 2013 35th International, Oct 2013, pp. 1-6.

[9] G. Lu, D. De, and W.-Z. Song, "Smartgridlab: A laboratory-based smart grid testbed," in Smart Grid Communications (SmartGridComm), 2010 First IEEE International Conference on, Oct 2010, pp. 143-148.

[10] D. Koss, D. Bytschkow, P. Gupta, B. Schatz, F. Sellmayr, and S. Bauereiss, "Establishing a smart grid node architecture and demonstrator in an office environment using the soa approach," in Software Engineering for the Smart Grid (SE4SG), 2012 International Workshop on, June 2012, pp. 8-14.

[11] M. Duchon, P. Gupta, D. Koss, D. Bytschkow, B. Schätz, and S. Wilzbach, "Advancement of a sensor aided smart grid node architecture," in The 3rd International Workshop on Smart Sensor Networks (IWSSN 2014), Shanghai, P.R. China, Oct. 2014.

[12] [Online]. Available: http://openweathermap.org/api

[13] [Online]. Available: http://openforecast.sourceforge.net/

[14] J. W. Taylor, "Triple seasonal methods for short-term electricity demand forecasting," European Journal of Operational Research, vol. 204, no. 1, pp. 139-152, 2010.

[15] L. Suganthi and A. A. Samuel, "Energy models for demand forecasting - a review," Renewable and Sustainable Energy Reviews, vol. 16, no. 2, pp. 1223-1240, 2012.

[16] [Online]. Available: http://www.rabbitmq.com/

[17] [Online]. Available: https://github.com/mysmartgrid/hexabus/

[18] [Online]. Available: http://www.sms-guard.org/

[19] "Daily real-time locational marginal pricing," 2013. [Online]. Available: http://www.pjm.com/markets-and-operations/energy/real-time/lmp.aspx 